\title{
Review of: "Inflammatory pathways amongst people living with HIV in Malawi differ according to socioeconomic status"
}

Rong Chen

Potential competing interests: The author(s) declared that no potential competing interests exist.

This is an interesting study that focuses on the relationship between the immune activation and socioeconomic status. In the better economic situation, PLWHs may have higher cfPWV, which means a higher risk of cardiovascular disease. In the low economic situation, PLWHs may have greater immune activation and endothelial damage, which is also the risk factor of non-communicable disease. This means that for PLWHs with different socioeconomic status, different methods should be taken to reduce the risk factors of non-communicable diseases. This can help people carry out targeted support policies and aid efficient allocation of limited health care resources.

There are two concerns with this study which include the following:

1. For people with better socioeconomic status, whether they have HIV or not, they may become a risk group of cardiovascular disease due to factors such as over nutrition and sedentary lifestyles. From this point, the management of chronic non communicable diseases should be the same in HIV patients and non HIV patients.

2. For people with low socioeconomic status, unsafe water, poor housing materials, low income and low education are not the direct risk factors for chronic non communicable diseases. The most direct impact of low socioeconomic status is helminth infection and recurrent bacterial infection. It is not clear whether the effects of these infectious diseases on immune activation can be deduced to increase the incidence of non infectious diseases. 